\title{
Incidental finding of incarcerated De Garengeot hernia during laparoscopic cholecystectomy
}

\author{
E. Hayakawa*, T. Hackett \\ Grand Rapids Medical Education Partners, Central Michigan University, Michigan, United States
}

Received: June 16, 2015

DOI: $10.5430 /$ css.v1n1p26
Accepted: July 19, 2015

URL: http://dx.doi.org/10.5430/css.v1n1p26

\begin{abstract}
The current case is of an 83-year-old female with vague abdominal pain found to have an incarcerated De Garengeot hernia during laparoscopic cholecystectomy with intraoperative cholangiogram. A review of the literature of this rare type of hernia will be presented in this case report.
\end{abstract}

Key Words: De Gerongeot, Hernia, Femoral, Appendix, Incarcerated

\section{INTRODUCTION}

An 83 year old female with nonspecific diffuse abdominal pain had multiple plausible causes of her complaints. These include a hiatal hernia, cholelithiasis with an elevated lipase, and an incarcerated De Garengeot's hernia. Like many other cases of De Garengeot's hernias, the patient presented atypically and was not diagnosed pre operatively. In fact, it was only discovered upon routine examination of her abdomen during laparoscopic cholecystectomy.

\section{Case presentation}

An 83-year-old female was referred by her primary care giver for surgical management of a presumed symptomatic hiatal hernia. The patient complained of diffuse abdominal pain with radiation to the back. She also mentioned some abdominal bloating and occasional nausea. In addition, the pain became worse with physical activity, but was not affected by the intake of food. Her initial work up included a CT scan which exhibited a large hiatal hernia with half the stomach above the diaphragm, but no obstruction. Laboratory values were obtained that did include a comprehensive metabolic panel, cbc, and lipase. She was found to have a lipase of 344 , which was consistent with a mild pancreatitis. Subsequently, an abdominal ultrasound was obtained that showed gallstones.

Patient's medical history included hypertension as well as atrial fibrillation for which she took multiple cardiac medications. Past surgeries were a hysterectomy and tonsillectomy. Family history was non-contributory. On initial physical exam, the patient's abdomen was soft, non-tender, nondistended, with present bowel sounds, and a well healed midline scar. No masses, organomegaly, or hernias in the groin were palpated.

The patient's clinical picture was the most consistent with ongoing symptomatic cholelithiasis with development of mild gallstone pancreatitis. Thus, a laparoscopic cholecystectomy with intraoperative cholangiogram was performed. At end of the procedure, routine inspection of the entire abdomen was implemented. It was at that time that an incarcerated femoral hernia was noted on the right side of her groin. Reduction of

\footnotetext{
*Correspondence: E. Hayakawa; Email: Emiko.hayakawa@grmep.com; Address: 205 Heron Drive NW, Apt 305B, Grand Rapids, MI 49534, United States.
} 
the hernia was attempted laparoscopically without success requiring conversion to an open repair. A transverse oblique incision was made in the right groin. No direct or indirect hernias were noted. The floor of the inguinal canal was incised and the pre peritoneal space was entered. The appendix was then visualized in the femoral space between Cooper's ligament and the inguinal ligament (see Figure 1). The appendix was overall normal in appearance. The sac containing the appendix was then reduced. The peritoneal cavity was entered and an appendectomy was performed. The defect was fixed using a standard McVay repair (see Figure 2). The patient tolerated procedure well and was discharged the following day. Upon follow up one month later, her symptoms had resolved. No further labs or workup for her hiatal hernia were obtained due to the resolution of her symptom.
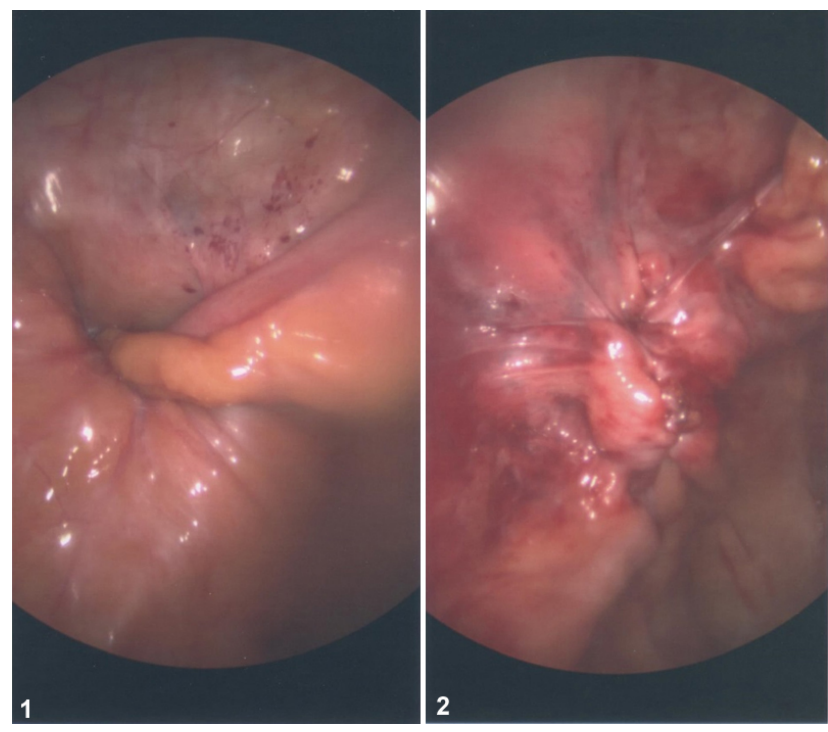

Figure 1. Appendix in femoral space prior to reduction

Figure 2. Hernia defect after McVay repair

\section{Discussion}

There are many types of hernias with different contents within their sac. The appendix is rarely found within a hernia sac. One of these types of hernias is an Amyand's hernia, where the appendix is found within an inguinal hernia. De Garengeot hernia, the presence of the appendix inside a femoral hernia is even rarer. This hernia was first named after the surgeon Rene Jacues Croissant De Garengeot in the 18 th century. It accounts for $0.5 \%-5 \%$ of all femoral hernias. Like the more common types of femoral hernia, the incidence of De Garengeot hernias are twice as high in females as in males. ${ }^{[1-5]}$

Most people with De Garengeot hernias present asymptomatically or may present with vague abdominal pain. According

Published by Sciedu Press to some authors, it is felt that appendicitis in a femoral sac can be a primary obstructive event or it can be secondary to the constriction of the appendix by the tight neck of the hernia sac. ${ }^{[5,6]}$ The largest case series found in the literature review is of 7 patients over a 15 year period. None were diagnosed pre operatively and the most common presenting symptom was painful groin swelling, which the present patient did not have. This series suggested repairing the hernia and performing an appendectomy based on what the appendix looks like within the sac. ${ }^{[5]}$ However, the decision to carry out an appendectomy on a De Garengeot hernia is more complex than the simple appearance of the appendix. The patient's age, co-morbidities, and the risk of any future surgical intervention has to be kept into consideration. The decision was made to perform an appendectomy due to the patient's age and co morbidities that would complicate a subsequent operation if needed.

Most of De Garengeot hernias are rarely diagnosed preoperatively as in this patient. Yet, in multiple case studies it appears CT is the most effective tool to utilize in order to make a diagnosis. CT findings would include intramural air density in an incarcerated hernia sac indicating intestinal involvement, but lack of obstruction or dilation of small bowel. ${ }^{[1,7]}$ In this case, the patient did have a CT performed, which showed a hiatal hernia, cholelithiasis, and diverticulosis. However, nothing was visualized within the right groin.

Although not intentionally utilized, many authors advocate laparoscopy as a safe and helpful adjunct when approaching hernias of unknown etiologies. Its use is especially emphasized when the groin hernias are atypical and the contents cannot be determined either clinically or radiologically. ${ }^{[8-10]}$ Another important point to be taken away from this case is that inspection of the entire abdomen and pelvis when doing laparoscopic procedures should be routine. It was especially important in this case because the patient's pain was nonspecific and could have been the result of multiple etiologies. Nevertheless, the patient's pain was most likely due to gallstones based on her symptoms and workup. However, missing the incarcerated femoral hernia on abdominal surveillance during surgery may have resulted in future complications such as appendicitis or the development of groin pain necessitating another surgery.

\section{Conclusion}

De Garengeot hernias are rare and are usually not diagnosed pre-operatively. Some case reports state the decision of whether to do an appendectomy should be based on how the appendix appears within the sac. However, the patient's age, co-morbidities, and presentation should be kept into 
consideration when making such a decision. This case reinforces the need to inspect the abdomen and pelvis at the time of surgery in order to reduce the risk of missing co-existing pathologies in the abdomen.

\section{REFERENCES}

[1] Ebisawa K, et al. Acute appendicitis in an incarcerated femoral hernia: a case of de Garengeot hernia. Case reports in gastroenterology.2009; 3(3): 313-317. PMid: 21103247. http://dx.doi.org/10.1159 1000250821

[2] Gabriel A, Alexander M. De Garengeot hernia: appendicitis within a femoral hernia. The American surgeon. 2005; 71(6): 526-527. PMid: 16044937.

[3] Gurer A, et al. Uncommon content in groin hernia sac. Hernia. 2006; 10(2): 152-155. PMid: 16172801. http://dx.doi.org/10.1007 /s10029-005-0036-4

[4] Isaacs Lawrence E, Chad Felsenstein H. Acute appendicitis in a femoral hernia: an unusual presentation of a groin mass. The Journal of emergency medicine. 2002; 23(1): 15-18. http://dx.doi.org /10.1016/S0736-4679(02)00455-9

[5] Sharma H, et al. De Garengeot hernia: an analysis of our experience. Hernia. 2007; 11(3): 235-238. PMid: 17340052. http: //dx.doi.org/10.1007/s10029-007-0208-5

[6] Sharma H, et al. Amyand's hernia: a report of 18 consecutive patients over a 15-year period. Hernia. 2007; 11(1): 31-35. PMid: 17001453. http://dx.doi.org/10.1007/s10029-006-0153-8

[7] Ash L, et al. Amyand's hernia: a case report of prospective CT diagnosis in the emergency department. Emergency Radiology. 2005; 11(4): 231-232. PMid: 16133611. http://dx.doi.org/10.1007 /s10140-005-0411-6

[8] Deeba S, et al. Laparoscopic approach to incarcerated and strangulated inguinal hernias. Journal of the Society of Laparoendoscopic Surgeons. 2009; 13(3): 327.

[9] Lin E, Wear K, Tiszenkel HI. Planned reduction of incarcerated groin hernias with hernia sac laparoscopy. Surgical Endoscopy And Other Interventional Techniques. 2002; 16(6): 936-938. PMid: 12163958. http://dx.doi.org/10.1007/s00464-001-8242-3

[10] Thomas B, et al. De Garengeot hernia. Journal of the Society of Laparoendoscopic Surgeons. 2009; 13(3): 455-457. 